\title{
DNA Polymerase Delta
}

National Cancer Institute

\section{Source}

National Cancer Institute. DNA Polymerase Delta. NCI Thesaurus. Code C16511.

A protein complex that has dual enzymatic activities in DNA synthesis. The enzyme complex both facilitates DNA synthesis by acting as a polymerase and exhibits exonuclease activity that degrades single stranded DNA in the $3^{\prime}$ to $5^{\prime}$ direction. It is a heterotetramer comprised of the DNA polymerase delta catalytic subunit and 3 DNA polymerase delta accessory subunits. 